\title{
A THz transverse electromagnetic mode two-dimensional interconnect layer incorporating quasi-optics
}

\author{
S. Coleman and D. Grischkowsky ${ }^{\text {a) }}$ \\ School of Electrical and Computer Engineering and the Center for Laser and Photonics Research, \\ Oklahoma State University, Stillwater, Oklahoma 74078
}

(Received 24 July 2003; accepted 12 September 2003)

\begin{abstract}
We report the demonstration of a planar $\mathrm{THz}$ interconnect layer capable of transmitting subpicosecond pulses in the transverse electromagnetic (TEM) mode over arbitrarily long paths with low absorption and no observable group velocity dispersion. Quasioptical elements are incorporated within the interconnect layer forming a configurable THz bandwidth TEM-mode planar interconnect with negligible group velocity dispersion and low loss. For a $146 \mathrm{~mm}$ guided path length, including four reflections, the pulses are broadened by the frequency dependent absorption of the interconnect layer from 0.28 to $0.32 \mathrm{ps}$, and attenuated by the factor 0.2. (C) 2003 American Institute of Physics. [DOI: $10.1063 / 1.1624474]$
\end{abstract}

Terahertz $(\mathrm{THz})$ waveguides have recently been demonstrated to be an alternative to high-speed coplanar transmission lines. ${ }^{1-4}$ For frequencies up to $3.5 \mathrm{THz}$, the attenuation coefficient of metal waveguides has been shown to be less than $1 / 10$ that of lithographically defined transmission lines on dielectric substrates. Within the passband of the waveguide, the measured power coupling into the waveguide was typically $40 \%$ of the incoming $\mathrm{THz}$ power. For the single conductor circular and rectangular metal waveguides the observed excessive $\mathrm{THz}$ pulse broadening was caused by the extreme group velocity dispersion (GVD) near the cutoff frequency. Such pulse broadening does not occur for the transverse electromagnetic (TEM) mode of a parallel-plate metal waveguide, since it has no cutoff frequency. The group and phase velocities of the TEM mode are determined solely by the dielectric. Recent experiments have shown efficient quasioptic coupling of freely propagating subpicosecond (subps) pulses of $\mathrm{THz}$ radiation into parallel-plate metal waveguides and the subsequent low-loss, single TEM mode propagation exhibiting negligible $\mathrm{GVD}^{3,4}$ Consequently, a $\mathrm{THz}$ interconnect, ${ }^{5}$ capable of propagating subps pulses with minimal loss and no distortion, has been realized.

However, all of these demonstrations involved quasioptical input and output coupling of freely propagating $\mathrm{THz}$ beams into and out of the $\mathrm{THz}$ waveguides. The remaining challenge is to efficiently connect the THz TEM waveguide with integrated circuitry. To address this problem we have begun to study a macroscopic planar interconnect approach using two-dimensional (2D) quasioptics. The interconnect consists of two relatively large (many centimeters) metal plates separated from each other by approximately $100 \mu \mathrm{m}$. Within this $100 \mu \mathrm{m}$ thick, free-space interconnect layer, planar quasioptical components are placed to guide, collimate, or focus the propagating THz TEM waves. This 2D planar quasioptics approach has the potential to realize a spatially localized point-to-point interconnect with the low-loss, broad bandwidth, and negligible GVD of the single-mode $\mathrm{THz}$ TEM planar metal waveguides.

${ }^{a)}$ Electronic address: grischd@ceat.okstate.edu
This 2D interconnect is related to previous works by Mink, in which he proposed and demonstrated the hybrid dielectric slab-beam waveguide (HDSBW), ${ }^{6,7}$ The HDSBW uses two distinct waveguiding principles to guide electromagnetic waves in a dielectric slab. In the direction normal to the slab the guided waves are described by the modes of the slab waveguide, while in the lateral direction the beams freely propagate and can be guided with quasi-optical components. In other work, spatiotemporal electro-optic imaging of propagating subps $\mathrm{THz}$ pulses, electro-optically generated by (fs) optical pulses in $0.5 \mathrm{~mm}$ thick nonlinear $\mathrm{LiNbO}_{3}$ crystals, was performed. ${ }^{8,9}$ For this case dielectric waveguides, diffractive, interferometric, and focusing elements were demonstrated for propagation lengths of several millimeters in $\mathrm{LiNbO}_{3}$.

Here we report an experimental demonstration of the validity of the $2 \mathrm{D}$ interconnect approach, by directing a well characterized beam of subps $\mathrm{THz}$ pulses into such a $2 \mathrm{D}$ interconnect layer and guiding the beam through the layer with four simple $2 \mathrm{D}$ mirrors. The $\mathrm{THz}$ pulses in the output beam from the 2D layer showed minimal broadening due to GVD, and their total attenuation coefficient was less than twice the attenuation coefficient of the corresponding planar copper waveguide. The minimal broadening of the output pulses was caused by their frequency dependent attenuation. This successful demonstration of 2D reflective quasioptics implies that more complex reflective optics should allow for confocal, integrated guided wave interconnect structures to be realized. In particular, with the incorporation of focusing optics, efficient point-to-point $\mathrm{THz}$ pulse communication within this interconnect plane should be possible.

The experimental setup of Fig. 1 consists of a photoconductively switched transmitter and receiver in the standard THz time domain spectroscopy configuration. ${ }^{1,4,5}$ In this arrangement a frequency dependent beam waist is present in the central confocal position. Here, a lens-interconnect-lens system is placed at this central position. The lenses are highresistivity silicon plano-cylindrical lenses of length $15 \mathrm{~mm}$ in the $x$-direction, $10 \mathrm{~mm}$ in the $y$-direction, with a $5 \mathrm{~mm}$ radius of curvature and a thickness of $6.56 \mathrm{~mm}$. At the focus of 


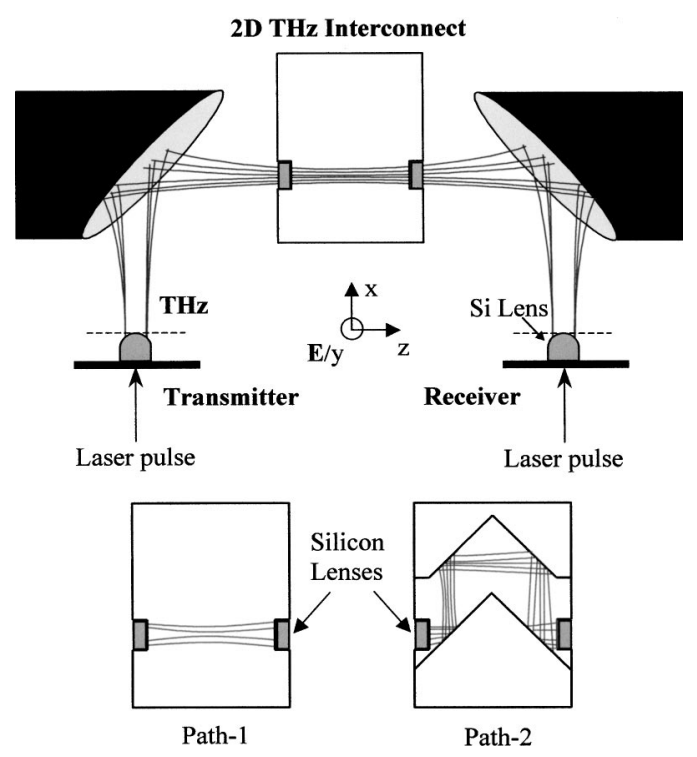

FIG. 1. Optoelectronic THz-TDS system incorporating either a $68 \mathrm{~mm}$ straight path (Path-1) or a $146 \mathrm{~mm}$ reflective path (Path-2) through the 2D interconnect layer.

each silicon lens the electric field is linearly polarized perpendicular to the interconnect plates, and a beam of elliptic cross section is produced. This beam has a frequency independent minor axis and a linearly frequency dependent major axis with $1 / e$ amplitude diameters of $200 \mu \mathrm{m}$ and $8 \mathrm{~mm}$, respectively, at $1 \mathrm{THz}$.

For this experiment two externally identical parallel plate interconnects were fabricated from a $9.5 \mathrm{~mm}$ thick oxygen free copper alloy 101 plate, machined to shape, and polished by hand. The external dimensions of each structure were $100 \mathrm{~mm}$ in the $x$-direction, $76 \mathrm{~mm}$ in the $z$-direction, with a $19 \mathrm{~mm}$ overall height in the $y$-direction. Both structures used $100 \mu \mathrm{m}$ copper alloy 110 shim stock to maintain the plate spacing, and the same material was used for the internal mirrors. The mirrors were hand polished such that the polished edges remained square with sharp corners and flat reflecting surfaces. The first interconnect incorporated no internal quasioptical elements and had a straight through path length of $68 \mathrm{~mm}$. This structure therefore functioned as a parallel-plate waveguide. The second interconnect had a $146 \mathrm{~mm}$ guided path length, achieved by making four reflections from planar quasioptical flat mirrors incorporated within the 2D interconnect layer. Because the two interconnect structures were externally identical, the positions of their included silicon lenses relative to the confocal beam waist of the THz-TDS system did not change with the internal THz path length.

The reference pulse, shown in Fig. 2(a), was taken with no interconnect or lenses present in the THz-TDS system. The propagated pulses through the $68 \mathrm{~mm}$ straight path and the $146 \mathrm{~mm}$ reflective path through the 2D interconnects are shown in Figs. 2(b) and 2(c), respectively. It should be noted that the two silicon cylindrical lenses of each interconnect reduce the transmitted signal by the multiplicative factor of 0.49 , owing to reflections from the high-index $(n=3.42)$ lens material. Each of the pulses shown is the result of a single measurement. The measured full width at half maximum (FWHM) pulsewidths of the propagated pulses through Downloaded 22 Sep 2004 to 141.14.235.78. Redistribution subject

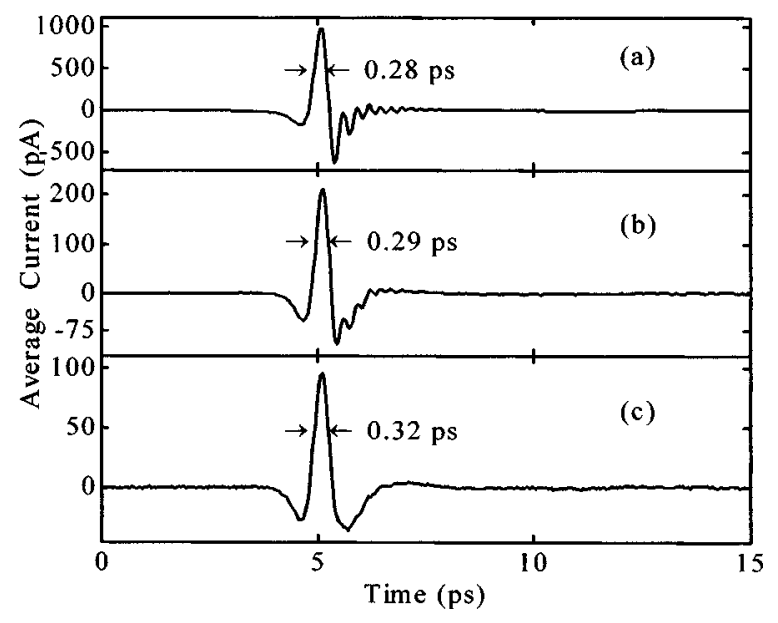

FIG. 2. (a) Reference pulse taken with neither lenses nor interconnect in the system. (b) Propagated pulse through the straight $68 \mathrm{~mm}$ path through the 2D interconnect. (c) Propagated pulse through the $146 \mathrm{~mm}$ reflective path through the interconnect.

the reference, the $68 \mathrm{~mm}$ straight path, and the $146 \mathrm{~mm}$ reflective path are $0.28,0.29$, and $0.32 \mathrm{ps}$, respectively. As reported in Ref. 4, we similarly observe no dispersive pulse broadening. The pulsewidths are only slightly broadened by the frequency dependent absorption, due to the finite conductivity of the copper plates and mirrors.

Figure 3 shows the relative amplitude spectra, scaled to facilitate comparison of the pulses. Each spectrum is the Fourier transform of a single time-domain scan. The FWHM of the amplitude spectra are $1.9 \mathrm{THz}$ for the reference, 1.2 $\mathrm{THz}$ for the $68 \mathrm{~mm}$ straight path, and $0.9 \mathrm{THz}$ for the 146 $\mathrm{mm}$ reflective path. The smoothness of the spectra with no observed low frequency cutoffs confirms TEM mode propagation.

The transfer function of the single-mode TEM planar waveguide based interconnect system can be written in the frequency domain as

$$
E_{\text {out }}(\omega)=E_{\text {ref }}(\omega) T C_{x} C_{y}^{2} R^{4} e^{-j\left(\beta_{z} L-\beta_{0} d\right)} e^{-\alpha L},
$$

where $E_{\text {out }}(\omega)$ and $E_{\text {ref }}(\omega)$ are the complex spectral components at angular frequency $\omega$ for the output (measured) and reference electric fields, respectively, $T$ is the amplitude

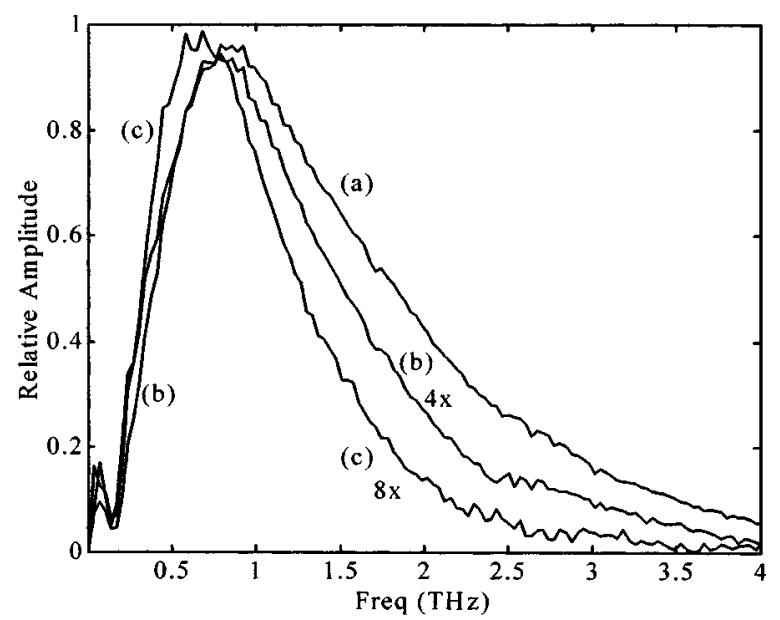

FIG. 3. Amplitude spectra of the propagated pulses of Figs. 2(a)-2(c), respectively. (a) Standard TDS system and (b) straight $68 \mathrm{~mm}$ path [spectrum multiplied by 4]. (c) $146 \mathrm{~mm}$ reflective path [spectrum multiplied by 8]. 


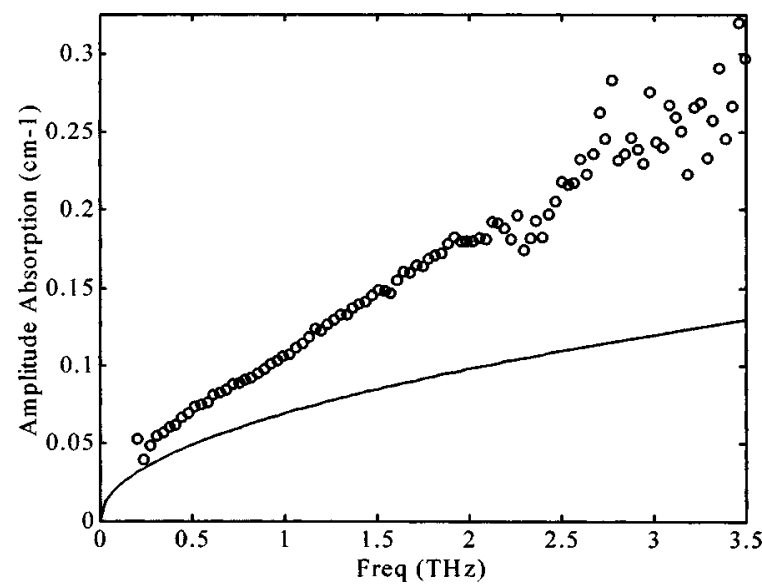

FIG. 4. Measured effective amplitude absorption $\alpha_{\text {eff }}$ (open circles) for the interconnect layer and the theoretical absorption $\alpha$ (solid line) for the TEM mode of a $100 \mu \mathrm{m}$ parallel plate copper waveguide.

transmission coefficient accounting for the Fresnel reflections from the cylindrical lens surfaces, $C_{x}$ is the coupling coefficient in the $x$-direction which is assumed to be $100 \%$ at the input and therefore is not squared. $C_{y}$ is the coupling coefficient in the $y$-direction. $R$ is the amplitude reflectivity of the $2 \mathrm{D}$ interconnect mirrors, set to unity for the straight path. $L$ is the guided wave path length in the interconnect and $d$ is the corresponding free space path length with the interconnect removed. $\alpha$ is the amplitude attenuation constant, $\beta_{z}$ is the phase constant, and $\beta_{o}=\left(2 \pi / \lambda_{0}\right)$, where $\lambda_{0}$ is the free space wavelength.

Applying Eq. (1) to the straight path (path-1) and reflective path (path-2) data separately and taking the absolute value of their complex ratio, we obtain an expression for an effective amplitude absorption coefficient $\alpha_{\text {eff }}$ that depends on the ratio of the measured electric fields, which include the effect of the coupling in the $x$-direction and the reflectivity of the four mirrors:

$$
\alpha_{\text {eff }}=\frac{1}{\left(L_{1}-L_{2}\right)} \ln \left|\frac{E_{\text {out } 2}}{E_{\text {out } 1}}\right| .
$$

The theoretical absorption coefficient for the TEM mode of a parallel plate waveguide is $\alpha=(10.88$ $\left.\times 10^{-3}\left[10^{7} / \sigma \lambda_{0}\right]^{0.5}\right) /\left(\eta_{0} b\right),{ }^{10}$ where $\eta_{0}$ is the wave impedance of free space $\left[\eta_{0}=\left(\mu_{0} / \epsilon_{0}\right)^{1 / 2} \sim 377 \Omega\right], b$ is the plate separation $(100 \mu \mathrm{m})$, and $\sigma$ is the conductivity of copper $\left(5.8 \times 10^{7} \Omega^{-1} \mathrm{~m}^{-1}\right)$. The measured effective absorption shown in Fig. 4 is the average of data taken from three separate experiments, using Eq. (2), with the theoretical absorption for a parallel plate waveguide plotted for comparison. This absorption is in reasonable agreement with previous waveguide data, ${ }^{3,4}$ even though it includes the four reflections and the $C_{x}$ coupling losses due to the nonconfocality of the system with the interconnect layer installed. As such, the reflectivity of the incorporated mirrors is considered to approach unity, thereby confirming the efficacy of this approach.
Compared to the minimum loss of the parallel-plate waveguide, the excess loss can be attributed to three mechanisms: First, the coupling coefficient $C_{x}$ was not removed from the measured data. In previous work the effect of this coupling was calculated based on a Gaussian $\mathrm{THz}$ beam and was removed. However, recent measurements show that our $\mathrm{THz}$ beams are not Gaussian. ${ }^{11}$ If a Gaussian beam were assumed, the absorption would be reduced by only 0.03 $\mathrm{cm}^{-1}$ at $2 \mathrm{THz}$. Second, we expect some loss as the structure likely has gaps of several microns between the plates and the mirror edges allowing leakage of the guided mode, although significant efforts were made to reduce this effect. Third, the skin depth of copper at the frequencies of interest is approximately $100 \mathrm{~nm}$, so that surface roughness equal to or greater than the skin depth may lead to an additional frequency dependent loss.

In conclusion, we have demonstrated a two-dimensional $\mathrm{THz}$ interconnect layer incorporating quasioptical mirrors. The mirrors have a minimal effect on the overall loss of the interconnect layer and TEM mode propagation is maintained for significant lengths after multiple reflections. This demonstration of planar reflective optics implies that more complex reflective optics should allow for fully confocal, integrated $\mathrm{THz}$ bandwidth interconnect structures to be fabricated. This technology allows for the possibility of guided wave $\mathrm{THz}$ bandwidth multiplexers, transmitter/receiver arrays, and densely integrated interconnect structures with common plates comprising the interconnect layer. Furthermore, the ability to focus within the interconnect layer should allow for intense subps pulses of $\mathrm{THz}$ to be achieved within the layer and guided wave based spectroscopy to be performed.

This work was partially supported by the National Science Foundation, the U.S. Army Research Office, and the Semiconductor Research Corporation, Center for Advanced Interconnect Systems Technologies.

${ }^{1}$ G. Gallot, S. Jamison, R. W. McGowan, and D. Grischkowsky, J. Opt. Soc. Am. B 17, 851 (2000).

${ }^{2}$ R. Mendis and D. Grischkowsky, J. Appl. Phys. 88, 4449 (2000).

${ }^{3}$ R. Mendis and D. Grischkowsky, Opt. Lett. 26, 846 (2001).

${ }^{4}$ R. Mendis and D. Grischkowsky, IEEE Microw. Wirel. Compon. Lett. 11, 444 (2001).

${ }^{5}$ D. Grischkowsky, IEEE J. Sel. Top. Quantum Electron. 6, 1122 (2000).

${ }^{6}$ J. W. Mink and F. K. Schwering, IEEE Trans. MTT 41, 1720 (1993).

${ }^{7}$ S. Zeisberg, A. Schuenemann, G. P. Monahan, P. L. Heron, M. B. Steer, J. W. Mink, and F. K. Schwering, IEEE Microw. Guid. Wave Lett. 3, 253 (1993).

${ }^{8}$ Nikolay S. Stoyanov, David W. Ward, Thomas Feurer, and Keith A. Nelson, Nature Materials 725, 95 (2002).

${ }^{9}$ Nikolay S. Stotanov, T. Feurer, David W. Ward, and Keith A. Nelson, Appl. Phys. Lett. 82, 674 (2003).

${ }^{10}$ N. Marcuvitz, Waveguide Handbook (Peter Peregrinus, London, U.K., 1993).

${ }^{11}$ M. T. Reiten, S. A. Harmon, and R. A. Cheville, J. Opt. Soc. Am. B 30, 2215 (2003). 